\title{
Bosons and Environment ${ }^{1}$
}

\author{
Enrico Celeghini* and Mario Rasetti ${ }^{\dagger}$ \\ * Dipartimento di Fisica and Sezione INFN \\ Università di Firenze, I50125 Firenze, Italy \\ ${ }^{\dagger}$ Dipartimento di Fisica and Unità INFM \\ Politecnico di Torino, I10129 Torino, Italy
}

\begin{abstract}
The role of the background in bosonic quantum statistics is discussed in the frame of a new approach in terms of coherent states. Bosons are indeed detected in different physical situations where they exhibit different and apparently unconnected properties. Besides Bose gas we consider bosons in particle physics and bosons in harmonic traps. In particle physics bosons are dealt with in a context where the number of observed particles is finite: here the relevant feature are the canonical commutation relations, which we shall show to be related to a Boltzmann-like distribution. A further case is Bose condensate in harmonic traps, where discrete spectrum leads us to predict, below $T_{c}$, a new critical temperature. The unified approach proposed shows that all differences can be ascribed to the environment.
\end{abstract}

\section{Introduction}

Bosons are discussed in different areas of physics with the implicit assumption that none of their properties is related to the characteristics of the Fock space where their states are defined (continuum or discrete) or to the number of particles (finite or, essentially, infinite). We discuss here different physical paradigms, all in Fock space and with unlimited occupation numbers, showing that such assumption is incorrect: different environments are not equivalent and unexpected relations with the background can be found.

We introduce first the algebraic approach to quantum statistics recently developed, showing that an equivalent but more powerful definition of bosons can be stated in terms of coherent states of the non compact algebra $s u(1,1)[1]$. In the necessary definition of consistent limit procedures for each physical problem, the analyticity properties imposed by group theory will be shown to play an essential role. The applications considered will deal with particle physics (PP), Bose gas, and Bose-Einstein condensation (BEC). In PP the interest is focused on canonical

1) Contributed Paper to Spin-Statistics Connection and Commutation Relations, R.C.Hilborn and G.M.Tino eds. (American Institute of Physics, New York, Sept. 2000) 
commutation relations (CCR), the energy spectrum is continuum and the number of particles is limited. The Bose gas is well exemplified by the blackbody radiation - once more with continuum spectrum but centered on Gibbs and Bose postulates and described in terms of finite density (and infinite number) of particles - and we consider it only to compare it with BEC where, together with the postulates, one has discrete spectrum and finite number of particles.

It will be shown that in PP bosons satisfy a Boltzmann-like statistics, and just because of that they are related to $h(1)$. There follows that they obey CCR, which are thus the correct commutation relations in this physical situation (even though, as we shall see, not in others). We shall then argue that the standard approach working for the blackbody, cannot be extended to BEC: in the latter for $T<T_{c}$, finite density approximation fails as the spectrum discreteness becomes relevant and a new physics emerges. In particular equipartition does not hold any longer and a new critical temperature is found, where the specific heat exhibits a spike.

\section{Algebras and Coherent States}

A relation between each statistics and a well defined algebra, has been found in 1998 [1] in terms of coherent states [2]. This has allowed us to select specific raising and lowering operators among those that one can arbitrarily introduce into Fock space. Bose-Einstein definition of bosons is indeed given by requiring the density matrix $\rho$ to be a multiple of the identity $\mathbf{I}$ on the Fock space $\mathcal{F}$, with no need of referring to any specific operator. One is then free to consider the operators $a^{ \pm}$ relating spaces with different number of particles $-\mathcal{F}_{n}$ and $\mathcal{F}_{n+1}-$ such that

$$
a^{+}|n\rangle=f(n+1)|n+1\rangle,
$$

where $f(n)$ is an arbitrary function. We can assume $f(n) \equiv \sqrt{n}$ but also e.g. $n$ or $e^{n}$. The choice of $a^{ \pm}$usually done - that derives from the Weyl-Heisenberg algebra $h(1)$ of position and momentum operators and implies CCR - is arbitrary or, more precisely, appears to be an independent postulate. In what follows such postulate will be shown, following [1], to be related to a specific quantum statistics even though, at first sight, an unsatisfactory one: only a careful discussion of the limit procedure (taking into account correctly the structure of the Fock space) clarifies that for bosons in the physical background of PP (and only in it) consistency holds.

We describe now briefly the procedure that leads from each algebra to its characteristic number of states of the system $W\{\bar{n}\}$; more details can be found in ref. [1]. Let us start from $h(1)$ (often considered as "the algebra" of bosons) and its coherent states. If we follow Condon-Shortley conventions, we have

$$
a^{+}|n\rangle=\sqrt{n+1}|n+1\rangle, \quad e^{a^{+}}|0\rangle=\sum \frac{1}{\sqrt{n !}}|n\rangle .
$$

If we disregard such conventions and do not fix any gauge, an arbitrary phase

(possibly a function also of an external parameter $t$, that we may assume to describe 
time) must be considered in front of each addendum of the coherent state, and the coefficients in the sum are exactly those needed to describe the 1-mode Boltzmann statistics (see e.g. [3]). Analogously, 2-modes are realized operating on the 2-mode vacuum $|0,0\rangle$ with $a_{1}{ }^{+}+a_{2}{ }^{+}$:

$$
e^{a_{1}++a_{2}+}|0,0\rangle=\sum \frac{\mathrm{e}^{i \phi\left(n_{1}, n_{2}, t\right)}}{\sqrt{n_{1} ! n_{2} !}}\left|n_{1}, n_{2}\right\rangle .
$$

In order to generalize to $M$-modes we have now only to consider the $M$-mode vacuum and the operator $\sum_{i=1}^{M} a_{i}{ }^{+}$(in a more formal language, we operate on the highest weight of the direct product representation with the iterated coalgebra $\left.\Delta^{M}\left(a^{+}\right)=\sum a_{i}^{+}[4]\right)$ :

$$
\exp \left[\Delta^{M}\left(a^{+}\right)\right]|0,0, \ldots, 0\rangle=\sum_{\{\bar{n}\}} \frac{\mathrm{e}^{i \phi(\bar{n}, t)}}{\sqrt{n_{1} ! n_{2} ! \ldots n_{M} !}}\left|n_{1}, n_{2}, \ldots, n_{M}\right\rangle .
$$

This is equivalent to

$$
W\{\bar{n}\} \propto \prod_{i} \frac{1}{n_{i} !}
$$

i.e. to the definition of Boltzmann statistics [3], that we thus obtain from the algebra $h(1)$ - which contains the canonical commutation relations $\left[\psi(x), \psi^{\dagger}(y)\right]=$ $\delta(x-y)$ as the relations $\left[a_{j}^{-}, a_{\ell}^{+}\right]=\delta_{j \ell}$ written in configuration space. Vice versa eq.(3) i.e. Boltzmann statistics can be rewritten as eq.(2) and thus requires $f(n)=$ $\sqrt{n}$ that implies $h(1)$ and its CCR.

We look now for an algebra, if any, related to Bose-Einstein statistics. It turns out to be sufficient selecting $f(n)=n$ to obtain

$$
\exp \left[\Delta^{M}\left(a^{+}\right)\right]|0,0, \ldots, 0\rangle=\sum_{\{\bar{n}\}} \mathrm{e}^{i \phi(\bar{n}, t)}\left|n_{1}, n_{2}, \ldots, n_{M}\right\rangle .
$$

Manifestly in this case all states in Fock space have equal probability of being occupied. Bose statistics appears thus to be related to $f(n)=n$. With this choice eq. (1) leads us to identifying $a^{+}$with the raising operator of the representation $\mathcal{D}_{1 / 2}^{+}$of $s u(1,1)$. Eq.(4) describes thus the $M$-mode coherent states of the $\left(\mathcal{D}_{1 / 2}^{+}\right)^{\otimes M}$ representation of $s u(1,1)$ (see [1]).

\section{Canonical Commutation Relations}

The results discussed in the previous section - surprising as they may sound relate on the one hand CCR to the Boltzmann statistics and on the other bosons to $s u(1,1)$. This apparently uncertain description signals indeed a more subtle and complex situation: bosons behave differently in different physical contexts. To 
demonstrate such thesis, we start from eq.(4) and we follow [3], collecting energy levels into cells, each containing $g$ levels. If cell $k$, of energy $\epsilon_{k}$, contains $N_{k}$ particles, then the number of states corresponding to the collection of occupation numbers $\{\bar{N}\}$ is given by

$$
W\{\bar{N}\} \propto \prod_{k} \frac{\Gamma\left(N_{k}+g\right)}{\Gamma(g) \Gamma\left(N_{k}+1\right)} .
$$

Eq.(5), that can be assumed as starting point of any bosons description in quantum statistics, is nothing but, as discussed in [1], the weight of the coherent state corresponding to the representation $\mathcal{D}_{g / 2}^{+}$of $s u(1,1)$. By construction, such weight provides the statistics of a finite number of bosons in Fock space with discrete spectrum. However, physical situations encountered in experiments (except for magnetic traps where BEC is currently investigated) are typically different. Appropriate limit procedures must thus be considered for eq.(5); these procedures, peculiar of each problem, must be included in the consistent definition of what bosons are in each physical situation.

In PP the spectrum is continuum (e.g. in momentum space) while the number of particles remains finite: the correct limit procedure on eq.(5) is thus given by the limit for $g \rightarrow \infty$ at $N_{k}$ fixed [5]:

$$
W\{\bar{N}\} \propto \lim _{g \rightarrow \infty} \prod_{k} \frac{\Gamma\left(N_{k}+g\right)}{\Gamma(g) \Gamma\left(N_{k}+1\right)}=\prod_{k} \frac{g^{N_{k}}}{N_{k} !} .
$$

Eq.(6) is exactly equivalent to eq.(3) (and to eq.(2)) from which it can be directly obtained with the same procedure of collecting elementary levels in cells that allows to obtain eq.(5) from eq.(4) [3]. This means that a finite number of bosons, in a continuum Fock space, satisfies Boltzmann's statistics and, thus, CCR. This result, which appears to contradict common wisdom, is actually consistent with all properties of bosons: all statistics - Bose, Boltzmann and Fermi - give the same distribution in these conditions as, in the physics of the continuum, only states with occupation numbers limited to zero and one have probabilities different from zero. The only statistical property that survives the limit is indeed the symmetry of the wave functions and the related Bose-Einstein correlation [6] [7].

\section{Bose-Einstein Condensation}

For the blackbody the limit is different: once more $g \rightarrow \infty$ (because the configuration space is continuum) but $N_{k} \rightarrow \infty$ as well (as blackbody is a theory of gases). Now the limit prescription is that the density remain finite: $0<N_{k} / g \equiv \delta_{k}<\infty$. In this way one recovers the usual formulas ${ }^{2}$.

2) One should not forget that the usual formalism is affected by a small but conceptually relevant difficulty: as for large energy $\delta \approx 0$, the limit prescriptions are not satisfied and a not zero probability is predicted for photons of energy greater than that of the entire blackbody. 
Let us now compare it with Bose-Einstein condensation in harmonic traps. A detailed technical discussion can be found in ref. [8]; we consider here only the aspects more closely related with statistics. As a finite fraction of particles migrate into the fundamental level, $\delta_{0} \approx \infty$ and the blackbody prescriptions are badly violated. In the standard approach, an ad hoc delta function is introduced in the density of states to allow for a macroscopic occupation of the ground state: a procedure which is not adequate in that it does not determine the ground state occupation. The theory of BEC for $T<T_{c}$ should be constructed on a different basis, namely not in terms of density but of $N$ - the total number of particles (finite) in a discrete spectrum. In particular the continuum approximation not only does not permit a rigorous treatment of the macroscopic single state occupation characteristic of BEC, but is far from the experimental situation, where typically $h \nu / k_{B} \approx 10 n K$ whereas the estimated temperature $T_{c} \approx 10^{2} n K$.

The solution to the problem can be found coming back to eq.(5), without any limit. We can thus easily look for the most probable distribution, maximizing the logarithm of $W\{\bar{N}\}$ (constrained by the two conditions $\sum_{i} N_{i}=N$ and $\sum_{i} \epsilon_{i} N_{i}=$ $E)$ with respect to all $N_{i}$ 's. One obtains in this way the exact equation for $N_{i}$ and $g$ finite:

$$
\psi\left(N_{i}+g\right)-\psi\left(N_{i}+1\right)=\alpha+\beta \epsilon_{i} \equiv h_{i}
$$

where $\alpha=\alpha(g)$ and $\beta=\beta(g)$ are the two Lagrange multipliers, clearly depending on the value of $g$, while $\psi$ is the digamma function [5].

For large argument $\psi$ coincides with the logarithm, thus for $N_{i} \gg 1$ and $g \gg 1$ it is easy to obtain the Bose-Einstein distribution we are well accustomed to:

$$
\delta=\frac{1}{e^{h}-1}
$$

but these limit assumptions do not hold either in PP (where the $N_{i}$ 's are finite) nor in harmonic traps (where both $g$ and the $N_{i}$ 's are finite). As the digamma function has poles for negative integers only, eq.(7) is defined almost everywhere in the complex plane and one could conjecture that it provides the solution for BEC. Unfortunately it is not so: in the presence of a physical 3-dimensional harmonic confining potential, levels are not degenerate (as different frequencies cannot be exactly equal) i.e. $g$ should be set equal to 1 , and this leads for eq.(7) to a perfectly acceptable but void equation (left hand side identically zero, implying $\alpha(1)=$ $\beta(1)=0$ ). The way out is found in the analyticity of group theory representations: while in the combinatorial approach $g$ is a multiplicity and thus an integer, in theory of representations it is simply a parameter that can assume any strictly positive real value. We can thus find by analytical continuation for $g \rightarrow 1$ the equation for not degenerate levels we need. $N_{i}$ results to be the solution of the implicit equation

$$
\psi^{\prime}\left(N_{i}+1\right)=\alpha^{\prime}+\beta^{\prime} \epsilon_{i} \equiv h_{i}^{\prime}
$$


where $\alpha^{\prime} \equiv \lim _{g \rightarrow 1} \alpha(g) /(g-1)$ and $\beta^{\prime} \equiv \lim _{g \rightarrow 1} \beta(g) /(g-1)$ are the new Lagrange multipliers to be determined by the constraints of fixed $N$ and $E$, and $\psi^{\prime}$ is the trigamma function [5], derivative of $\psi$. Eq.(9) is one-to-one and can be inverted, giving us the correct formula for discrete spectrum, to be used instead of eq.(8):

$$
N_{i}=\left[\psi^{\prime}\right]^{-1}\left(h_{i}^{\prime}\right)-1 \quad \text { for } \quad h_{i}^{\prime}<\pi^{2} / 6 \quad \text { and } \quad N_{i}=0 \quad \text { for } \quad h_{i}^{\prime} \geq \pi^{2} / 6,
$$

$\left[\psi^{\prime}\right]^{-1}$ denoting the inverse function of $\psi^{\prime}$ and $\pi^{2} / 6 \equiv \psi^{\prime}(1)$. The discrete description can now be implemented as follows: one assigns first the values of $N$ and $E$; from these the two Lagrange multipliers $\alpha^{\prime}=\alpha^{\prime}(N, E), \beta^{\prime}=\beta^{\prime}(N, E)$ are then obtained imposing the constraints with $N_{i}$ given by the defining equation (10); finally, inserting $\alpha^{\prime}(N, E)$ and $\beta^{\prime}(N, E)$ in (10) itself, one gets $N_{i} \equiv N_{i}\left(\alpha^{\prime}, \beta^{\prime}\right)=$ $N_{i}(N, E)$.

By inspection, the two formulas (8) and (10) exhibit a similar structure, because they have a pole with residue 1 for $h=0$ and they go to zero for large values of the argument. As condensation derives from such a behavior, combined with the feature that the number of states increases quadratically with energy, both descriptions are correct in predicting the collapse of the atoms in the fundamental level, also if the continuous approach is actually unable to describe what happens for $T<T_{c}$. The subtlety here is that the very concept of temperature, as defined in the theory of gases, cannot be straightforwardly extended to the condensate.

For $E / N$ large $\left(T>T_{c}\right.$, classical region) continuum quantum statistics should predict indeed for the second Lagrange multiplier $\beta^{\prime} \propto(E / N)^{-1}$, while we find $\beta^{\prime} \propto(E / N)^{-4}$. Also for $T$ large the energy is therefore not proportional to the inverse Lagrange multiplier $\beta^{\prime-1}$ but to the more complex expression $\left(\pi^{2} / 6-\alpha^{\prime}\right) \beta^{\prime-1}$. Temperature can then no longer be obtained, as for a gas, by $T=\left(k_{B} \beta^{\prime}\right)^{-1}$ : the whole scheme (including the very definition of temperature and its relation with energy, i.e. equipartition) must be reconsidered.

In order to obtain the temperature we resort to the more basic notion of entropy, adopting its Shannon information theoretical definition:

$$
S=-k_{B} \sum p_{i} \ln p_{i}
$$

where the $p_{i}$ 's are functions of $N$ and $E: p_{i} \equiv N_{i}(N, E) / N$. At fixed $N$ we thus straightforwardly obtain $S=S(E)$. Numerical elaborations finally give the temperature

$$
T \equiv T(E)=\left[\frac{\partial S}{\partial E}\right]_{N}^{-1}
$$

Once more this function is one-to-one, and from $T=T(E)$ we can obtain $E=E(T)$, whence the specific heat $C \equiv \frac{1}{N}\left[\frac{\partial E}{\partial T}\right]_{N}$ derives by (numerical) differentiation (see Fig. 1). 
The result can be checked for $T>T_{c}$, where $C$ is found to be constant, equal to $3 k_{B}$ (as it should, since the system does not condense and classical theory works). The physics is completely different for $T<T_{c}$ : $C$ is almost zero (as entropy and energy decrease with quite different slopes because only a few atoms progressively migrate from the warmer tail of the spectrum into the fundamental level) for $T_{1 / 2}<$ $T<T_{c}$, and it exhibits a peak for $T \approx T_{1 / 2}$, where condensation becomes a global effect. Note that after the spike, below $T_{1 / 2}$, the specific heat goes to zero as $T$ goes to zero, as required by Nernst's theorem. These results (as well as the complete description of the physics of BEC given in [8]) are quantitatively correct up to the detailed structure of the ground state, and this approach can perhaps be the appropriate background in which to introduce a collective perturbation [9].

In conclusion, the relevance of the algebraic structure of quantum statistics has been stressed. An important ingredient of the latter is analyticity that has been explicitly introduced in the scheme. This leads to a unified vision of the problem of bosons. Thanks to such approach, apparent differences of bosons in various contexts (particle physics, non-interacting gas, condensate) have been shown to be related only to the role of the environment. The notion of boson acquires thus a primary fundamental role in a unified vision which encompasses different definitions and embraces different fields of physics.

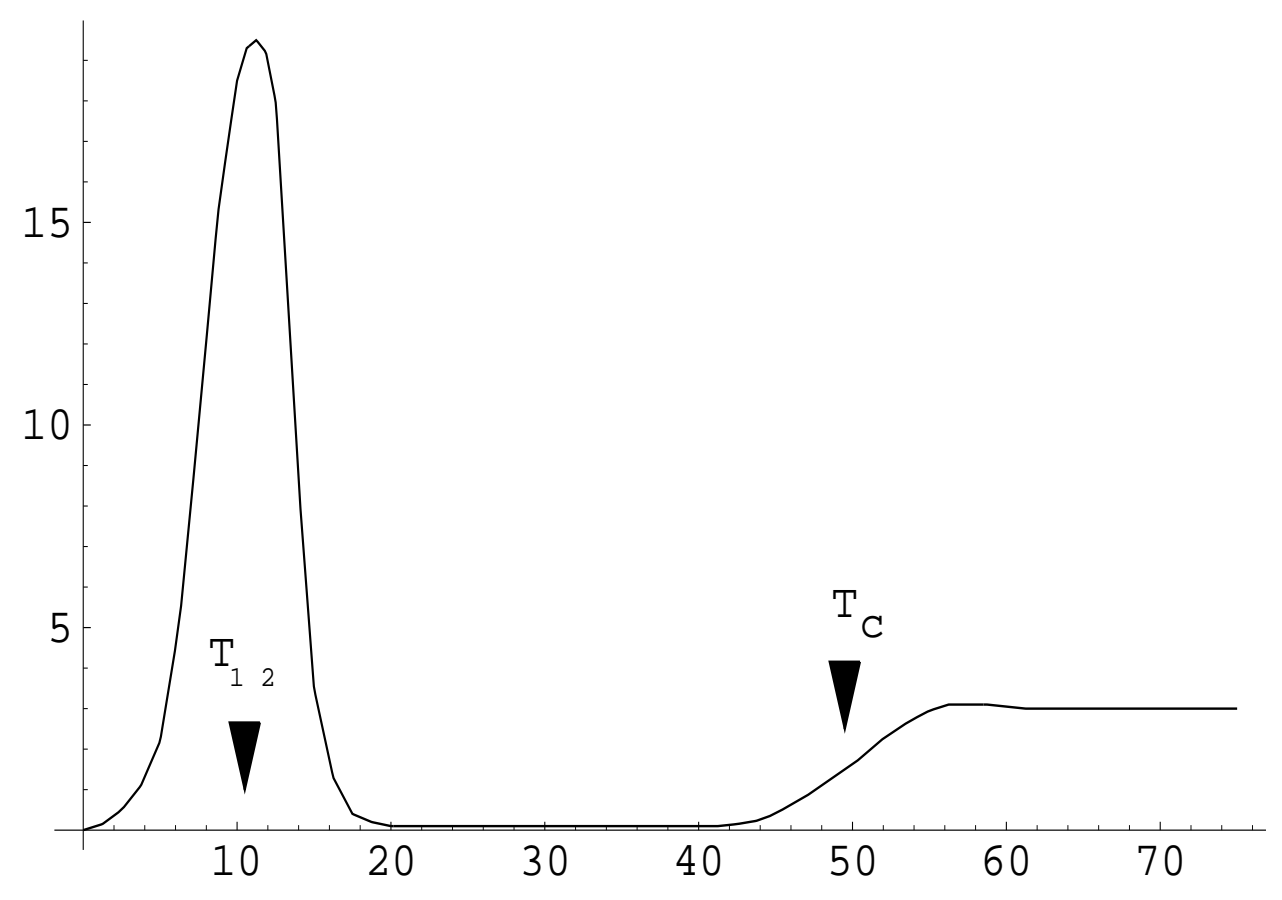

FIGURE 1. Specific heat $C$ vs. $T$ in units $k_{B}=h \nu=1$ for $N=10^{6}$ bosons in an isotropic harmonic trap, as predicted by discrete quantum statistics. 


\section{REFERENCES}

1. Celeghini E., and Rasetti M., Phys. Rev. Lett. 80, 3424 (1998).

2. Perelomov A. M., Generalized Coherent States and their Applications (SpringerVerlag, Berlin, 1986).

3. Huang K., Statistical Mechanics (J.Wiley \& Sons, New York, 1987).

4. Abe E., Hopf Algebras (Cambridge Tracts in Math. 74, Cambridge Un. Press, Cambridge, 1980).

5. Abramowitz M., and Stegun I., Handbook of Mathematical Functions (Dover, New York, 1965).

6. Lörstad B., Int. J. Mod. Phys. A4, 2861 (1989).

7. ALEPH Collaboration, Phys. Lett. B478, 50 (2000).

8. Celeghini E., and Rasetti M., arXiv:cond-mat/0004096, submitted to Phys. Rev. Lett.

9. F. Dalfovo, S. Georgini, L.P. Pitaevskii and S. Stringari, Rev. Mod. Phys. 71, 463 (1999). 